\title{
Link Budget Analysis for 5G Communication in the Tropical Regions
}

\author{
Trilochan Patra $\mathbb{D}^{1}$ and Swarup Kumar Mitra $\mathbb{D}^{2}$ \\ ${ }^{1}$ Techno International Newtown, Rajarhat, Newtown kol-700156, India \\ ${ }^{2}$ MCKV Institute of Engineering, Liluah, Howrah 711204, India \\ Correspondence should be addressed to Trilochan Patra; trilochanpatra266@gmail.com
}

Received 7 October 2020; Revised 11 November 2020; Accepted 9 December 2020; Published 24 December 2020

Academic Editor: Daniele Pinchera

Copyright (c) 2020 Trilochan Patra and Swarup Kumar Mitra. This is an open access article distributed under the Creative Commons Attribution License, which permits unrestricted use, distribution, and reproduction in any medium, provided the original work is properly cited.

\begin{abstract}
In the tropical regions, when a signal is transmitted from a transmitter to a receiver, the signal gets highly attenuated because of heavy rainfall. By using different diversity techniques, this signal attenuation is minimized. The multiple-input multiple-output (MIMO) system is an antenna diversity technique, and in this paper, this technique has been applied to the proposed communication link model designed for $5 \mathrm{G}$ communication in the tropical regions. Here, a proposed link budget has been set down to enhance the signal power and signal-to-noise ratio (SNR) at the receiver. In this paper, an experimental data sheet has also been adopted to achieve the desired result of the proposed link budget. In fine, a comparison of assumption values of the proposed link budget with practical values obtained from the experimental data sheet has been displayed in the result analysis part.
\end{abstract}

\section{Introduction}

Heavy rainfall is one of the main characteristics of the tropical regions. Because of this geographical phenomenon, the people of those regions always face a lot of obstacles like signal attenuation and rainfall detection in their spheres of communication. In [1], in order to inquire into the effect of rainfall on the dispersion of millimeter waves, the actual measurements have been executed at $26 \mathrm{GHz}$ frequency. The measurements are obtained by applying a microwave $5 \mathrm{G}$ radio connection system for $1.3 \mathrm{~km}$ path length carried out at Universiti Teknologi Malaysia Johor Bahru, Malaysia. At the same time, the enumeration received from the measurement for the most bad month is lesser than that of the ITU-R (International telecommunication union) model. The main effect of rainfall on electromagnetic wave propagation has produced a number of concentrated studies which focus on the attenuation of rain. These studies have been conducted to enquire and foretell the attenuation of rain in various areas for different frequency bands. The previous studies were carried out by Robertson and King [2], Mueller [3], Wexler and Weinstein [4], and Anderson et al. [5]. In the work executed by Mueller [3], the actual ratings were designed to test for long distance rainfall reduction by $0.62 \mathrm{~cm}(\mathrm{~cm})$. From that study, it was discovered that average rainfall of $0.62 \mathrm{~cm}$ produced $0.6 \mathrm{~dB} / \mathrm{mile} / \mathrm{mm} / \mathrm{hr}$ via a oneway connection. In the same year 1946, another measurement study was conducted by Robertson and King in Bell Mobile Laboratories in New York [2]. He studied the impact of rain on the spread of $1.09 \mathrm{~cm}$ wavelength for the zone between $1 \mathrm{~cm}$ and $4 \mathrm{~cm}$. It turned out that there was greater than $25 \mathrm{~dB}$ reduction that led to each mile in the rain cloud ratings. In addition, the rainfall waves more than $3 \mathrm{~cm}$ in light and the average rainfall was reduced, but it was possible to reach $5 \mathrm{~dB}$ per mile within cloudburst. In 1947 , another measurement study on rainfall reduction of $1.25 \mathrm{~cm}$ was produced by Anderson et al. in the United States Navy Electronics Labs in California [5]. That experimental setup contained a maximum 6400 feet optical path length and nine units of rainfall measurements placed in the same area. Both patterns of unvarying and varying rainfall were used in the region of the test system. From that study, that light was recognized as rainfall balance which could diminish normal contact link without rain by about $10 \%$. As a result, many measurement 


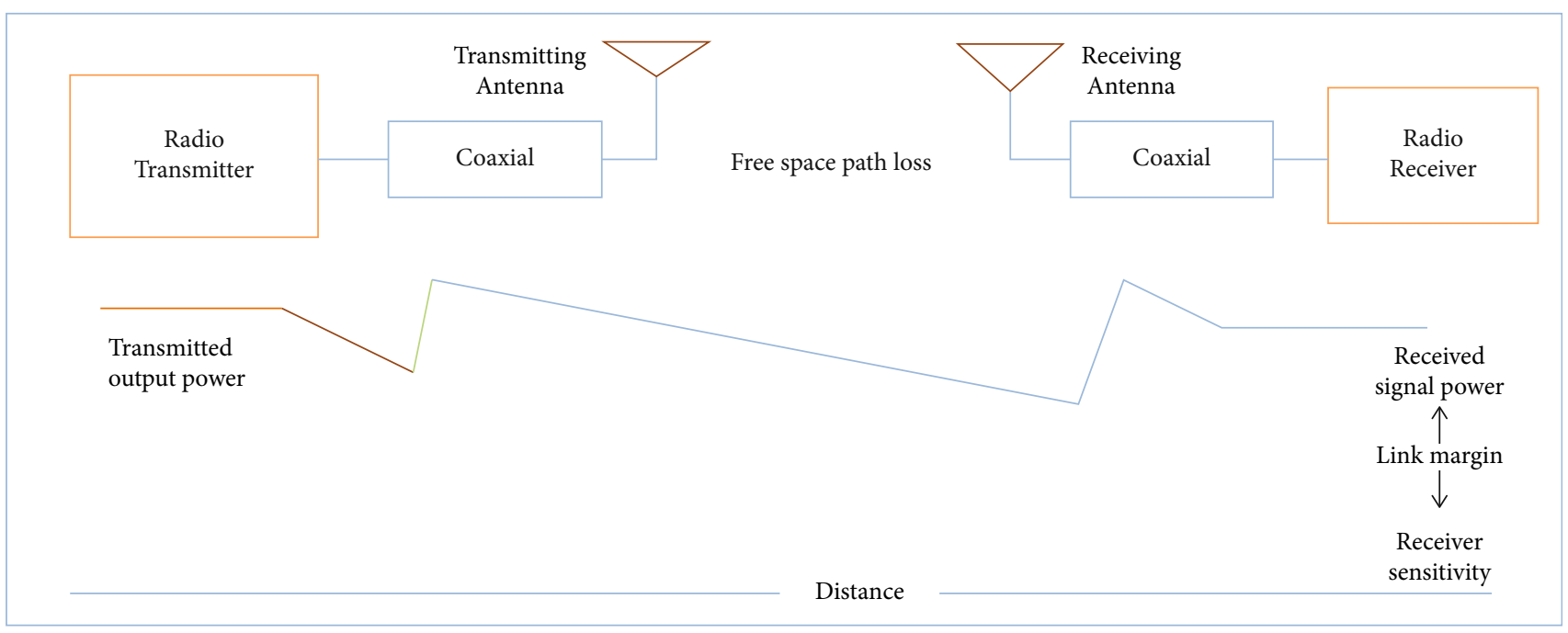

Figure 1: Power transmission in wireless system through free space.

studies are being rapidly developed throughout the world. In Malaysia, the first study was started which focused on the attenuation of rain in addition to wireless communication system and this study was implemented in the early 1990s at Universal Transverse Mercator (UTM) [6] and Universiti Sain Malaysia (USM) [7]. In these studies, the actual measurements were found in different places and had different frequency bands. Many researchers and developers have been exerting their best efforts for a long time through $5 \mathrm{G}$ communication to overcome the obstacles caused by heavy rainfall in the tropical regions, and many budgets have also been set down to remove these obstructions. According to $28 \mathrm{GHz}$ MIMO $(4 \times 4)$ channel computation for LOS and NLOS, the capacity of the channel and link budget of the system are analyzed in [8]. The capacity of the MIMO channel is simulated as a distance function. The main objective in [9] is to find out the interdependence of the frequency of path loss of the signal for various environments and for different ranges between the terminals of the antennas for foretelling the subsequent wireless canal for appropriate designs. In [10], the work relates to the prediction of the theory and the assessment of end-to-end, slow-moving, and fastmoving forms, as well as the total loss of pathway that occurs at an urban international radio station with the various rise of the station and transmitting subscriber antenna in terms of roofing. The basic properties of $\mathrm{THz}$ waves have been investigated in [11], and the availability of high-quality data for $\mathrm{THz}$ wireless links is obtained. In [12], an example link budget has been described. In [13], a close inspection of the characteristics of mmWave dispersion, channel designing, and suggestion of device such as structure and application of antenna device for mmWave including link budget of the system have been introduced into presence. But, no link budget appears to be reflected in the aforesaid papers for the benefit of tropical regions using MIMO technique. So, a link budget of the proposed communication link model has been put forward in this paper using MIMO technique for $5 \mathrm{G}$ communication for the tropical regions. Thereafter, with a view to increasing signal power as well as signal-to-noise ratio (SNR) at the receiver, this proposed link budget which depends on different parameters has been placed in this paper.

In this paper, in this proposed communication link model $4 \times 4$, MIMO technique has also been used. The multiple-input multiple-output (MIMO) technique multiplies the capacity of channels to increase the signal power at the receiver and also to enhance the reliability of the system [14-16]. Multiplexing gain is also an advantage of MIMO technique as multiple data streams from different transmitting antennas are transferred to different receiving antennas at the uniform frequency [17]. As the MIMO technique has been applied to this proposed model, a brief description of measurements of MIMO systems has been depicted in Section 2.

As the proposed budget in this paper relates to communication link, it is necessary to mention some features of the communication link. For wireless connectivity, link pointing provides an easy connection between the transmitter and receiver. At this basic link, the transmitted signal passes along the line-of-sight route to the receipient and medium is the free space. The purpose of the communication link is to check the signal-to-noise ratio (SNR) at the receiver to verify the performance of a link. When the signal strength is reduced by geometric spread of the front wave, it is generally known as free space loss. The signal strength is still distributed over the front wave, the area increases as the distance from the transmitter increases. As a result, the density of the power decreases. With free space link, if Friis figure is introduced, it becomes possible to calculate the loss of path. The power transmission in a wireless system through free space is shown in Figure 1.

Section 1 in this paper denotes only the introduction portion. Section 2 displays the measurement of the MIMO system. Section 3 depicts the proposed communication link model. Section 4 describes the link budget of the proposed model. Section 5 illustrates the link budget using experimental data. Section 6 exhibits the result analysis part. Section 7 relates to conclusion part and in fine, references are quoted. 


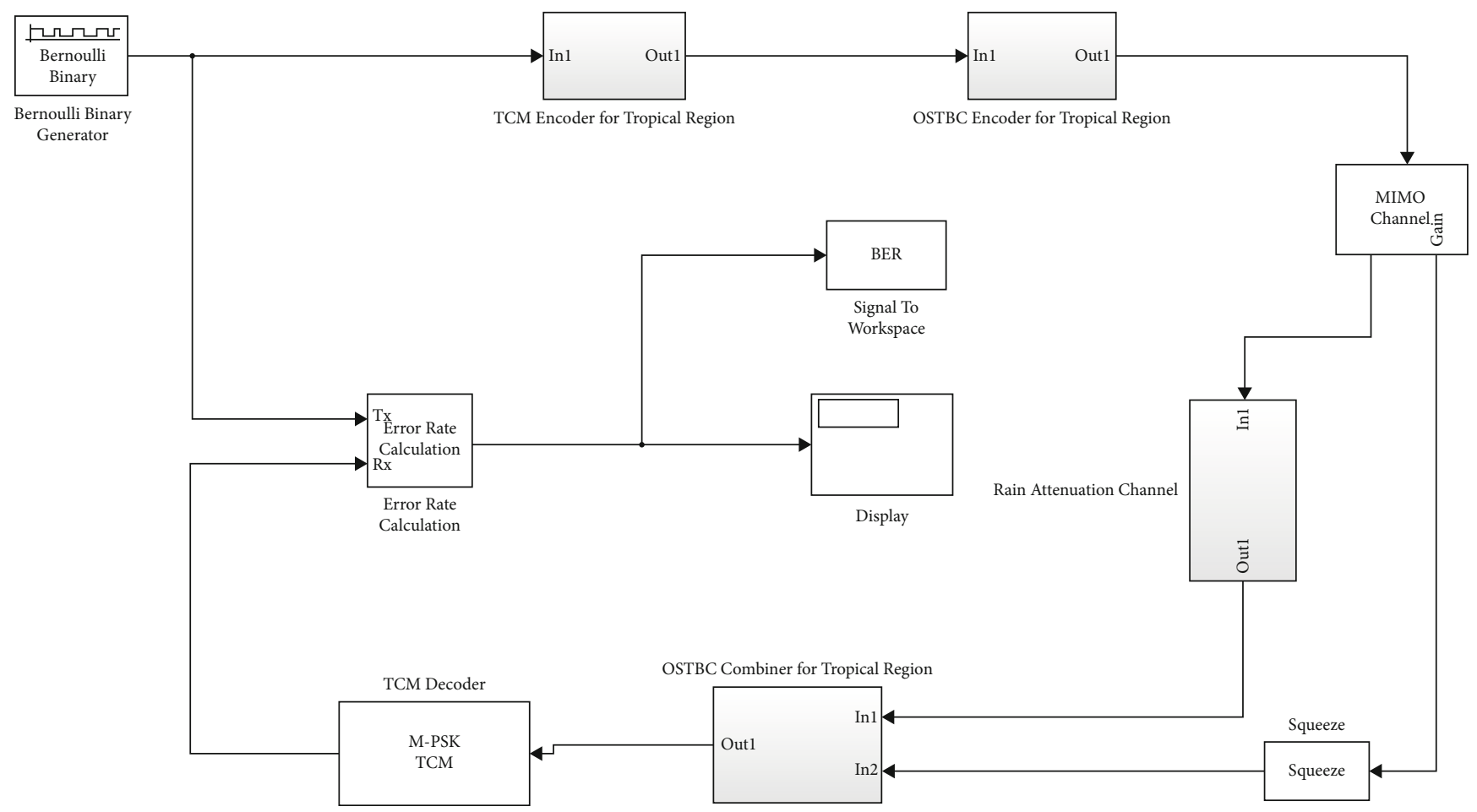

FIgure 2: Communication link model for $5 \mathrm{G}$ communication in the tropical regions.

\section{Measurements of MIMO System}

The MIMO transfer system uses a number of transfer methods and host data transmission antennas across the channel. The number of transmitting antennas is noted by $U$ and the number of receiving antennas is noted by $V$. The gradually changing frequency flat fading channels have been considered to emulate the model of MIMO transmission as $p=W x+y$ [18], where $p$ is the $V \times 1$ average vector, $x$ is the $U \times 1$ transmitted complex value vector, $W$ is $U \times V$ complex-valued channel matrix, and $y$ is the addition of $V \times 1$ which is a complex-valued white Gaussian noise vector (AWGN) with variation $\sigma_{\mathrm{y}}{ }^{2}$. Now, the unlinked branch resources have been considered having equal power $P_{\mathrm{ST}}$ and again it is presumed that all the receiving antennas get the identical average received power $P_{\mathrm{AR}}$. The acceptable level of signal-to-noise ratio (SNR) obtained at single receiving antenna is $\gamma^{\prime}=P_{\mathrm{AR}} / \sigma_{\mathrm{y}}{ }^{2}$. For convenience, variables are defined as $M=\max (V, U)$ and $N=\min (V, U)$. For presumptions, the MIMO capacity is written as [19].

$$
\mathrm{Cm}=\sum_{j=1}^{N} \log _{2}\left(1+\frac{\gamma^{\prime}}{V} \lambda j\right) \frac{\text { Bits } / \mathrm{s}}{\mathrm{Hz}}
$$

Here, $\lambda j$ is the $j$ th eigenvalue of $Z$, which is reflected as

$$
Z= \begin{cases}H_{e} H_{e}^{H}, & V \leq U, \\ H e^{H} H e, & V \geq U,\end{cases}
$$

where $H_{e}^{H}$ indicates the Hermitian transpose of the $H_{e}$ matrix. Equation (1) displays that a MIMO system comprises $N$ parallel single-input single-output (SISO) channels, where each channel possesses gain $\lambda j$.

\section{Description of the Proposed Model}

The 5G network connection model is devised to fix the proposed link budget as shown in Figure 2. The input samples are produced from the Bernoulli binary generator as the vector output. Input samples now pass through a trellis coded modulation (TCM) encoder designed for tropical regions. Automatic gain control (AGC) is added to the TCM encoder. This encoder is formed in such a way that when the signal passes through this state of encoding, the signal strength remains high. After that, the input samples pass across the orthogonal space time block code (OSTBC) encoder devised for tropical regions. This encoder installs an input message using OSTBC. This encoder is devised in such a way that when the input message passes through this encoder, its signal-to-noise ratio (SNR) remains high. The input signal now goes through the MIMO channel. The MIMO channel filters the input signal through the withering MIMO multipath channel. In the MIMO channel, there are two types of decaying channels viz. Rayleigh fading channel and Rician fading channel. From the MIMO station, the signal transits through the rain mitigation station. When the signal passes through this rain attenuation channel, the signal power decreases. Now, this input signal makes its way through the OSTBC combiner. This combiner mixes the available input signal and channel limitations according to the OSTBC 
TABle 1: Parameters of the proposed link budget.

\begin{tabular}{lccc}
\hline & Assumption 1 & & \multicolumn{2}{c}{ Assumption 2 } & Parameters & Values \\
Parameters & Values & Distance $(\mathrm{m})$ & 400 \\
\hline Distance $(\mathrm{km})$ & 1.6 & Frequency $(\mathrm{GHz})$ & 24.5 \\
Frequency $(\mathrm{GHz})$ & 71.5 & Transmitted output power $(\mathrm{dBm})$ & 18.5 \\
Transmitted output power $(\mathrm{dBm})$ & 16 & Transmitting antenna gain $(\mathrm{dBi})$ & 42.5 \\
Transmitting antenna gain $(\mathrm{dBi})$ & 41 & Receiving antenna gain $(\mathrm{dBi})$ & 42.5 \\
Receiving antenna gain $(\mathrm{dBi})$ & 41 & Background noise $(\mathrm{dBm} / \mathrm{Hz})$ & -174 \\
Background noise $(\mathrm{dBm} / \mathrm{Hz})$ & -174 & Bandwidth $(\mathrm{MHz})$ & 748 \\
Bandwidth $(\mathrm{MHz})$ & 26 & Noise figure at receiver $(\mathrm{dB})$ & 3 \\
Noise figure at receiver $(\mathrm{dB})$ & 4 & Loss for rain $(\mathrm{dB})$ & 27 \\
Loss for rain $(\mathrm{dB})$ & 30 & Link margin $(\mathrm{dB})$ & 35 \\
Link margin $(\mathrm{dB})$ & 20 & &
\end{tabular}

structure. Therefore, the input signal passes along the TCM decoder. This decoder uses the Viterbi algorithm to determine trellis-coded fluctuation data which are organized using a phase-shift method. Thereafter, the input signal goes through the error rate calculation which includes the error rate of the received data as compared to the delayed version of the transmitted data. Block output is a three-dimensional vector that contains an error rating, followed by the number of errors found and the total number of symbols compared. This vector is transmitted to the workspace or output port.

\section{Link Budget of the Proposed Model}

A budget of a link is a way to measure the effectiveness of a link. The power received from a wireless connector is determined by three factors viz. power transmission, gain of transmitting antenna, and gain of receiving antenna. If that power which removes the loss of free space for the communication link is greater than the minimum accepted signal level of the received radio, a link is possible. The difference between the minimum signal obtained and the actual received power is called the link margin. The amount of link margin required is reduced by the use of reduction techniques such as antenna variation or frequency repetition. A simple link budget equation is reflected as per following:

$$
\begin{aligned}
\text { Power received in } \mathrm{dB}= & \text { Power transferred in } \mathrm{dB} \\
& + \text { gains in } \mathrm{dB}-\text { losses in } \mathrm{dB} .
\end{aligned}
$$

If it is considered that the antennas are adjusted for maximum transmission and reception, in free space,

$$
\text { Prs }=\frac{\text { GtsAerPts }}{4 n r^{2}}
$$

where Aer is the aperture of the receiving antenna. Since Aer $=\mathrm{Grs} \lambda^{2} / 4 n, \lambda$ is the wavelength:

$$
\text { Prs }=\text { GrsGtsPts }\left[\frac{\lambda}{4 n r}\right]^{2} .
$$

TABle 2: Assumption values of SNR for assumption 1 and assumption 2 from Table 1 .

\begin{tabular}{lccc}
\hline Assumptions & $\begin{array}{c}\text { Received signal } \\
\text { power }(\mathrm{dBm})\end{array}$ & Noise power $(\mathrm{dBm})$ & SNR $(\mathrm{dB})$ \\
\hline Assumption 1 & -65.618 & -95.827 & 30.209 \\
Assumption 2 & -35.774 & -82.238 & 46.464 \\
\hline
\end{tabular}

TABLE 3: MIMO channel capacity calculation for assumption values.

\begin{tabular}{lcc}
\hline Assumptions & SNR(dB) & MIMO Channel capacity(bits/s/Hz) \\
\hline Assumption 1 & 30.229 & 19.860 \\
Assumption 2 & 46.464 & 22.154 \\
\hline
\end{tabular}

Now, the link budget equation including all the loss and gain effects is expressed logarithmically and is written in the following manner [16]:

$$
\text { Prs }=\text { Pts }+ \text { Gts }+ \text { Grs }- \text { Lts }-\mathrm{Lpf}-\mathrm{Lml}-\mathrm{Lrs},
$$

where Prs is the power received $(\mathrm{dBm})$, Pts is the output power transmitted $(\mathrm{dBm}), \mathrm{Gts}$ is the transmitting antenna gain $(\mathrm{dBi})$, Grs is the receiving antenna gain $(\mathrm{dBi})$, Lts is the losses of a transmitter ( $\mathrm{dB})$, Lpf is the path loss of free space $(\mathrm{dB}), \mathrm{Lml}$ is the losses of different kinds which includes losses for body, fading margin, rain, polarization mismatch, etc.) $(\mathrm{dB})$, and Lrs is the losses of a receiver $(\mathrm{dB})$.

Losses produced out of propagation between the antennas of the transmitter and receiver, usually referred to as the path loss, are illustrated in dimensionless form with the help of normalization of the distance to the wavelength:

$$
\operatorname{Lpf}(\mathrm{dB})=20 \log _{10}\left[4 n\left(\frac{D}{\lambda}\right)\right]
$$

where the distance $D$ and wavelength $\lambda$ are alike units.

In some cases, it is easy to assume losses separately because of distance and wavelength, but in that case, it is 
TABLE 4: Parameters used for the experiment in the tropical regions.

\begin{tabular}{lccc}
\hline Parameters & K-band & E-band & E-band \\
\hline Frequency & $21.8 \mathrm{GHz}$ & $73.5 \mathrm{GHz}$ & $73.5 \mathrm{GHz}$ \\
Distance & $1.8 \mathrm{~km}$ & $1.8 \mathrm{~km}$ & $300 \mathrm{~m}$ \\
Bandwidth & $28 \mathrm{MHz}$ & $750 \mathrm{MHz}$ & $750 \mathrm{MHz}$ \\
Type of antenna & Directional $0.6 \mathrm{~m}$ & Directional $0.3 \mathrm{~m}$ & Directional $0.2 \mathrm{~m}$ \\
Polarization type & (ANT3 $0.623 \mathrm{HP})$ & (ANT2 $0.380 \mathrm{HP})$ & $($ ANT2 $0.280 \mathrm{HP})$ \\
Antenna gain & Vertical & Vertical & Vertical \\
Antenna half power beam width & $40.7 \mathrm{dBi}$ & $46.5 \mathrm{dBi}$ & $43.5 \mathrm{dBi}$ \\
Maximum transmitted output power & 1.7 (degree) & $0.8($ degree $)$ & $1.1($ degree $)$ \\
\hline
\end{tabular}

important to keep track of the applied units, as each option involves a different offset. An example of loss because of distance $(\mathrm{km})$ is displayed below [20]:

$$
\operatorname{Lpf}(\mathrm{dB})=92.45 \mathrm{~dB}+20 \log [F(\mathrm{GHz})]+20 \log [D(\mathrm{~km})]
$$

where $D$ is the distance in $\mathrm{km}$ and $F$ is the frequency in $\mathrm{GHz}$.

Now, to calculate the SNR values, it is inevitable to calculate the noise power at the receiver. The receiver thermal noise is expressed by the following equation [16]:

$$
P_{t n}=K_{b} T_{k} B_{w}
$$

where $P_{t n}$ is the receiver thermal noise power in watts, $K_{b}$ is Boltzmann's constant, $T_{k}$ is the receiver temperature at Kelvin, and $B_{w}$ is the bandwidth in $\mathrm{Hz}$. below:

So, the noise power is mathematically expressed as [8]

$$
N_{p}=P_{t n}+N_{s e} F
$$

where $N_{s e} F$ is the noise figure. To set down the proposed link budget for the proposed model, the necessary parameters and their assumption values are shown below in Table 1 [21-23]. For considering parameters and their assumption values, it is necessary to know how with the increase of operating frequency rain attenuation increases but this phenomenon depends on the average rain rate per annum. For computing rain rate and rain attenuation, a valid and reliable data sheet is required which is available in [1].

Now using Equations (6), (8), (9), and (10) the values of received signal power and noise power have been computed for assumption 1 and assumption 2. The SNR values have been calculated by subtracting the noise power from received signal power. The calculation of SNR values is shown mathematically by the following equation:

$$
\mathrm{SNR}=\operatorname{Prs}-N_{p}
$$

The calculated values of received signal power, noise power, and SNR have been tabulated and demonstrated in Table 2 .
Using Equation (1), the capacity of the MIMO channel applied to this proposed model has been calculated for assumption 1 and assumption 2 and these values are given in Table 3.

\section{Link Budget Using Experimental Data}

For link budget analysis, the signal-to-noise ratio computation arrangement and test site atmosphere are discussed in the following manner.

For this experiment, the transmission equipment and receiver equipment of the K-band and E-band for this rating campaign have been provided by Ericsson, namely, Mini Links ML-6363 and ML-6352. The details of specification for ML-6363 and ML-6352 are depicted in [24, 25].

21.8 GHz K-band and 73.5 GHz E-band setups have been in use since September 2018 to study the effect of rainfall. The $21.8 \mathrm{GHz}$ connector has been fixed to support up to $225 \mathrm{Mbps}$ via a $28 \mathrm{MHz}$ bandwidth channel switching and flexibility up to 1024 QAM. The $73.5 \mathrm{GHz}$ connector has been designed to support up to $4.532 \mathrm{Gbps}$ through a $750 \mathrm{MHz}$ channel bandwidth and flexible mode switching up to 256 QAM. Average parameters of the setting of the above links including specifications are given in Table 4 where both the transmitter and receiver in each link accept the same types of antennas. Data importer receiver records the maximum and minimum amount of reference signal level regularly at a 15-minute interval. Both links are available at the University of Technology Malaysia (UTM), Kuala Lumpur (KL), Malaysia, by radio length of line-of-sight road of $1.8 \mathrm{~km}$. Transmitter for both links with the label Site A (3 $11^{\prime} 7^{\prime \prime} \mathrm{N}, 10143^{\prime} 46^{\prime \prime} \mathrm{E}$ ) are available on the top of a five-story student residence (49 meters above sea level) outside the university campus.

A receiver for both links with the site named $\mathrm{B}\left(310^{\prime} 21^{\prime \prime} \mathrm{N}\right.$, $10143^{\prime} 10^{\prime \prime} \mathrm{E}$ ) is found at level 16 in one of the upper administrative buildings ( 43 meters above sea level) at the university campus.

Another E-band link was established within UTM, KL campus whose assesment system parameters are listed in Table 4. The receiver is located on site $\mathrm{B}$ while transmitter labeled as site C (300 m LOS from site B) $\left(310^{\prime} 20^{\prime \prime} \mathrm{N}, 101\right.$ $\left.43^{\prime} 19^{\prime \prime} \mathrm{EE}\right)$ is located on the top of the UTM Revenue House building (41 meters above sea level) [26]. 
TABLE 5: SNR values obtained from the experimental data sheet.

\begin{tabular}{|c|c|c|c|c|}
\hline Frequency & Distance & Received signal power $(\mathrm{dBm})$ & Noise power $(\mathrm{dBm})$ & SNR $(\mathrm{dB})$ \\
\hline K-band $(21.8 \mathrm{GHz})$ & $1.8 \mathrm{~km}$ & -52.924 & -95.447 & 42.523 \\
\hline E-band (73.5 GHz) & $1.8 \mathrm{~km}$ & -56.880 & -81.168 & 24.288 \\
\hline E-band $(73.5 \mathrm{GHz})$ & $300 \mathrm{~m}$ & -47.317 & -81.168 & 33.683 \\
\hline
\end{tabular}

TABLE 6: MIMO channel capacity calculation for experimental data.

\begin{tabular}{lcc}
\hline Frequency band & SNR (dB) & $\begin{array}{c}\text { Channel capacity } \\
\text { (bits/s/Hz) }\end{array}$ \\
\hline K-band $(21.8 \mathrm{GHz}$, distance $1.8 \mathrm{~km})$ & 42.523 & 21.776 \\
E-band $(73.5 \mathrm{GHz}$, distance $1.8 \mathrm{~km})$ & 24.288 & 18.410 \\
E-band $(73.5 \mathrm{GHz}$, distance $300 \mathrm{~m})$ & 33.683 & 20.297 \\
\hline
\end{tabular}

In this experiment, three link setups have been established. To collect rain rate, data of the aforesaid three link setups, three HOBO (Honest Observer by Onset) data recording rain gages has been inserted in the channel path. The data recording the rainfall system is powered by a battery and contains a data recorder with a light rainfall gauge of $0.2 \mathrm{~mm}$ sensitivity. Details of definition of a rain gauge are provided in [27]. Two rain gages have been inserted at the receiver site $(\mathrm{Rx})$ while the third is placed at the transmitter (Tx) site. Two acceptable receiver rain gages (called $R_{G} R x$ and $R_{G} R x 1$ ) have been placed on the top of the two separate buildings but the distance of the receiver from one building is $130 \mathrm{~m}$ and that of the receiver from the other building is $380 \mathrm{~m}$. Again, a transmitter rain gage $\left(\mathrm{R}_{\mathrm{G}} \mathrm{Tx}\right)$ is placed on the top the building near the transmitter building which is approximetly 50 meters from the transmitting antenna. The rainfall is recorded by the rain gages for every minute interval.

Now, using Equation (6), (8), (9), and (10), the received signal power, noise power, and SNR values at the receiver for experimental data have been calculated. These calculated SNR values for practical data are shown in Table 5.

Now, using Equation (1), the MIMO channel capacity for the above experimental data has been calculated and demonstrated in Table 6.

\section{Result Analysis}

From Tables 2 and 5, it is observed that the assumption SNR values for assumption 2 reflect higher than the practical values obtained from the experimental data sheet. The SNR values depend on received signal power and noise power. The received signal power and noise power depend on various parameters. Here, only six parameters viz. distance, frequency, transmitted output power, transmitting or receiving antenna gain, MIMO channel capacity, and bandwidth have been chosen for the proposed link budget. Now, six figures (Figures 3-8) have been plotted below to show how the received signal power, noise power, and SNR values vary with the aforesaid parameters. From Figures 3 and 4, it is seen that the received signal power values are diminished when the

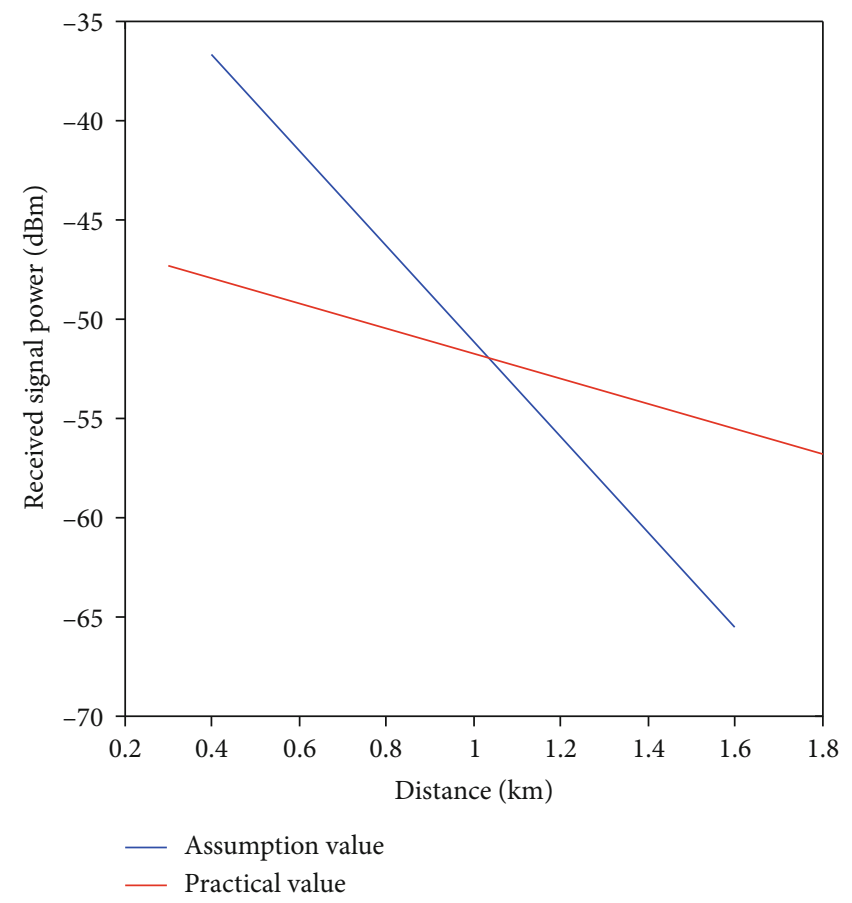

FIgURE 3: Variation of received signal power with distance.

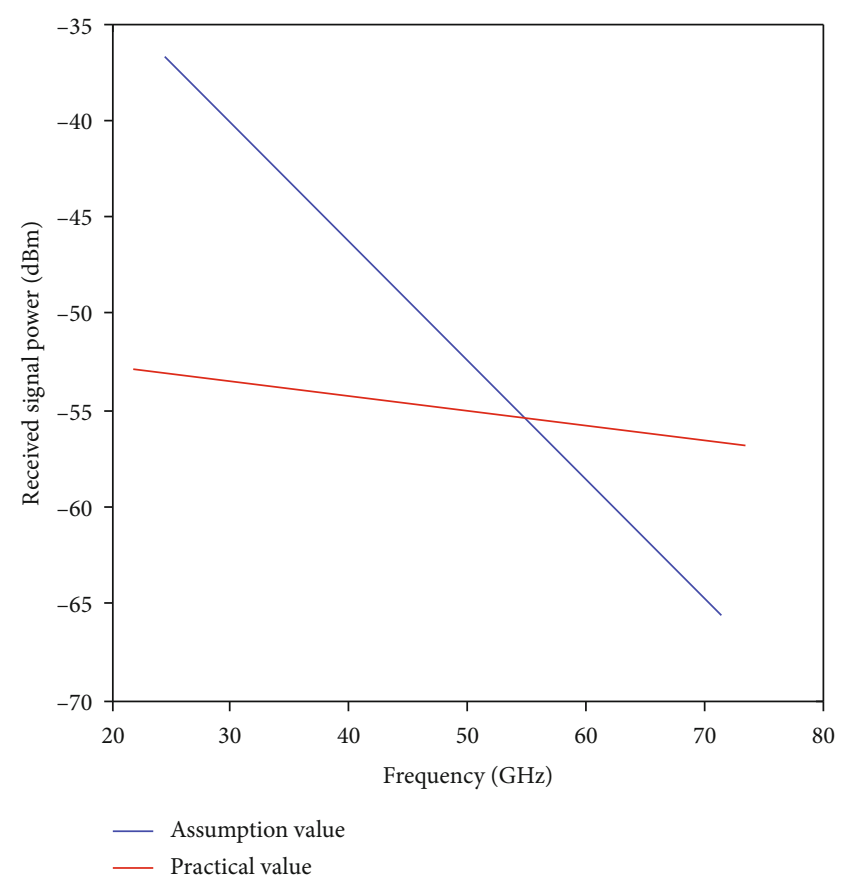

FIGURE 4: Variation of received signal power for frequency. 


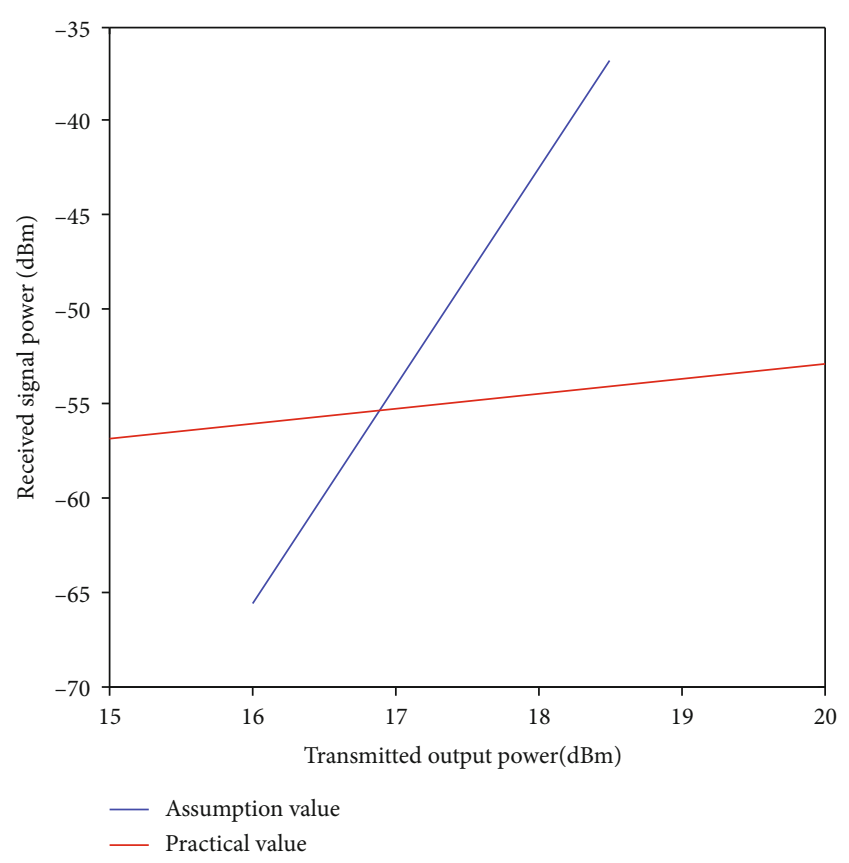

FIGURE 5: Variation of received signal power with transmitted output power.

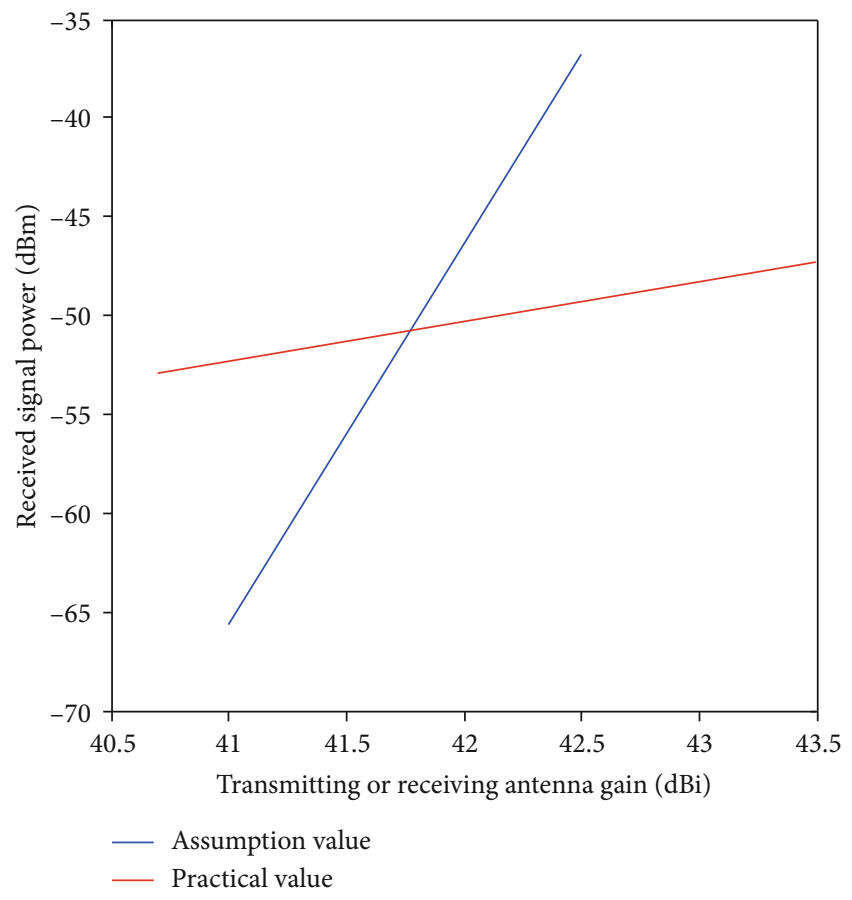

FIGURE 6: Variation of received signal power for transmitting or receiving antenna gain.

distance and frequency are enhanced. From Figures 5 and 6, it is observed that the received signal power values increase with the increase of transmitted output power and transmitting or receiving antenna gain. From Figure 7, it is noticed that the noise power is enhanced with the enhancement of bandwidth. From Figure 8, it is observed that the SNR values

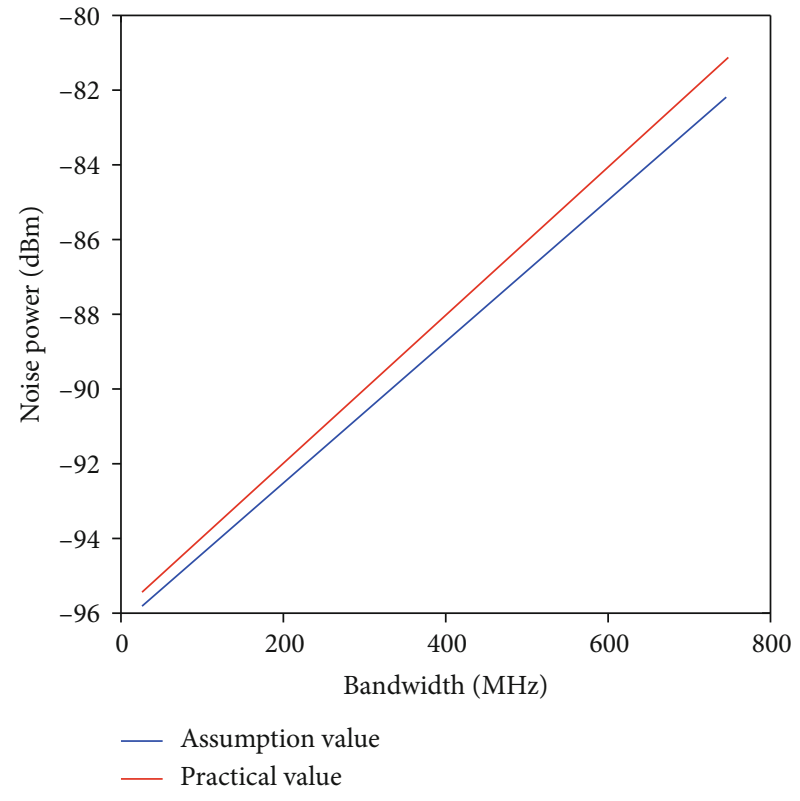

Figure 7: Variation of noise power for bandwidth.

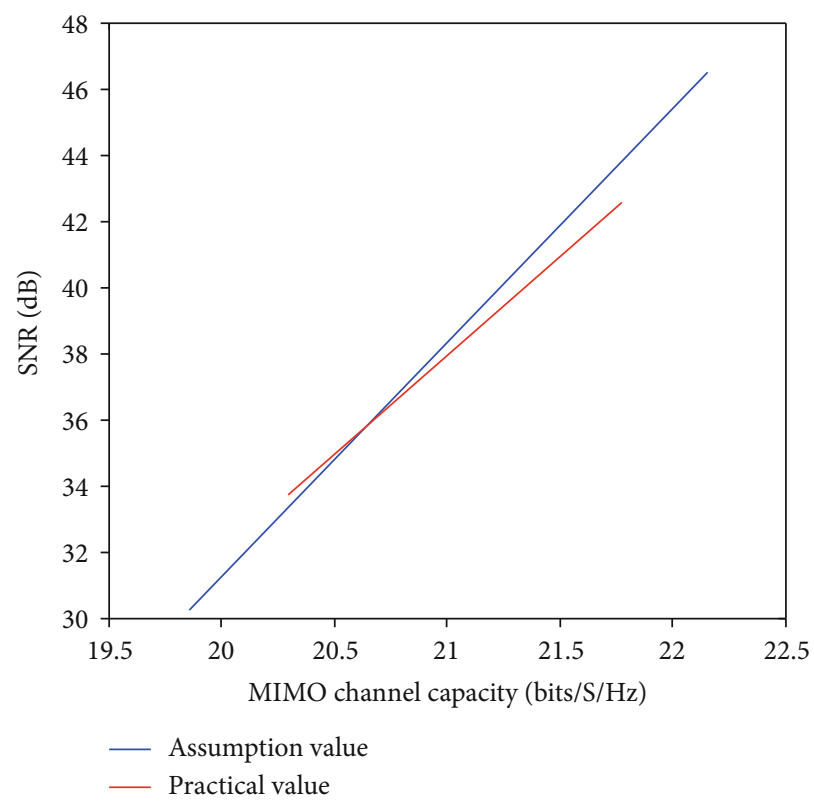

FIgURE 8: Variation of SNR for channel capacity.

are increased with the increase of the MIMO channel capacity.

\section{Conclusions}

In this paper, a link budget of a proposed communication link model has been laid down. From Table 2, it is observed that the SNR value for assumption 2 is $46.464 \mathrm{~dB}$ which seems most befitting to obtain a reliable communication link. From Figures 3-6, it would transpire that the received signal power values depend on the distance, frequency, transmitted output power, and transmitting antenna gain or receiving 
antenna gain. From Figure 8, it is observed that the SNR values depend on the MIMO channel capacity. From Figure 7 , it is seen that the noise power value depend on the bandwidth. The received signal power values increase for lower frequency and minimal distance. The received signal power values are also enhanced for higher values of transmitted power, transmitting antenna gain, and receiving antenna gain. The SNR values increase with the increase of the MIMO channel capacity. The noise power increases with the increase of bandwidth. The SNR values depend on received signal power and noise power. From Figures 5-8, it is observed that the assumption values of the proposed link budget provide better result than that of practical values obtained from the experimental data sheet. From Figures 3 and 4 , it is observed that the assumption values reflect better result than that of practical values for lower distance and lower frequency. In Figures 3-6, the assumption values and practical values reflect far relationship as the assumption values render better result than the practical values. A reliable link budget is obtained for lower frequency, minimal distance, minimal bandwidth, higher values of transmitted output power, higher values of transmitting antenna gain, higher values of receiving antenna gain, and higher values of MIMO channel capacity. So, from the overall work performed in this paper, it is concluded that the proposed link budget is highly expected to heighten the reliability of $5 \mathrm{G}$ communication in the field of wireless communication for the tropical regions. As a result, the people of these regions will get the benefit of obtaining a high-quality message signal in their domain of communication.

\section{Data Availability}

For practical verification, an experimental data sheet has been included in this paper and this data sheet is collected from Ericsson, namely, Mini Links ML-6363 and ML-6352. The details of specification for ML-6363 and ML-6352 are given in $[24,25]$ of this paper. This data sheet is demonstrated in Table 4 of this paper.

\section{Conflicts of Interest}

The authors declare that they have no conflicts of interest.

\section{References}

[1] I. Shayea, T. A. Rahman, M. H. Azmi, and M. R. Islam, "Real measurement study for rain rate and rain attenuation conducted over $26 \mathrm{GHz}$ microwave $5 \mathrm{G}$ link system in Malaysia," IEE Access, vol. 6, pp. 19044-19064, 2018.

[2] S. D. Robertson and A. P. King, "The effect of rain upon the propagation of waves in the 1- and 3-centimeter regions," Proceedings of the IRE, vol. 34, no. 4, pp. 178p-180p, 1946.

[3] G. E. Mueller, "Propagation of 6-millimeter waves," Proceedings of the IRE, vol. 34, no. 4, pp. 181p-183p, 1946.

[4] R. Wexler and J. Weinstein, "Rainfall intensities and attenuation of centimeter electromagnetic waves," Proceedings of the IRE, vol. 36, no. 3, pp. 353-355, 1948.
[5] L. J. Anderson, J. P. Day, C. H. Freres, and A. P. D. Stokes, "Attenuation of 1.25-centimeter radiation through rain," Proceedings of the IRE, vol. 35, no. 4, pp. 351-354, 1947.

[6] "Final Reports on Rain Attenuation Studies for Communication Systems Operating in Tropical Regions, Wireless Communication Research Laboratory, Universiti Teknologi Malaysia," 2000.

[7] A. Yagasena and S. I. S. Hassan, "Worst-month rain attenuation statistics for satellite-Earth link design at Kuband in Malaysia," in 2000 TENCON Proceedings. Intelligent Systems and Technologies for the New Millennium (Cat. No.00CH37119), pp. 122-125, Kuala Lumpur, Malaysia, 2000.

[8] A. Xia, Z. Wang, S. Geng, X. Zhao, R. Zhang, and Y. Liu, “28 GHz MIMO channel capacity analysis for $5 \mathrm{G}$ wireless communication systems," in 2018 12th International Symposium on Antennas, Propagation and EM Theory (ISAPE), Hangzhou, China, December, 2018.

[9] N. Blaunstein and Y. Ben-Shimol, "Prediction of frequency dependence of path loss and link-budget design for various terrestrial communication links," IEEE Transactions on Antennas and Propagation, vol. 52, no. 10, 2004.

[10] N. Yarkoni, N. Blaunstein, and D. Katz, "Link budget and radio coverage design for various multipath urban communication links," Radio Science, vol. 42, no. 2, 2007.

[11] T. Schneider, A. Wiatrek, S. Preussler, M. Grigat, and R. Peter Brau, "Link budget analysis for terahertz fixed wireless links," IEEE Transactions on Terahertz Science and Technology, vol. 2, no. 2, 2012.

[12] T. Cella, P. Orten, and T. Ekman, "Design of a practical and compact mm-wave MIMO system with optimized capacity and phased arrays," International Journal of Antennas and Propagation, vol. 2014, Article ID 608345, 9 pages, 2014.

[13] I. A. Hemadeh and M. E. Hajjar, "Millimeter-wave communications: physical channel models, design considerations, antenna constructions and link- budget," IEEE Communications Surveys and Tutorials, vol. 20, no. 2, pp. 870-913, 2018.

[14] A. Arvanitis, G. Anagnostou, N. Moraitis, and P. Constantinou, "Capacity study of a multiple element antenna configuration in an indoor wireless channel at $60 \mathrm{GHz}$," in Proceedings of the IEEE 65th Vehicular Technology Conference (VTC'07), pp. 609-613, China, 2007.

[15] I. Sarris and A. Nix, "Design and performance assessment of maximum capacity MIMO architectures in line-of-sight," IEE Proceedings - Communications, vol. 153, no. 4, pp. 482488, 2006.

[16] D. Gesbert, H. B. Olcskei, D. A. Gore, and A. J. Paulraj, "Outdoor MIMO wireless channels: models and performance prediction," IEEE Transactions on Communications, vol. 50, no. 12, pp. 1926-1934, 2002.

[17] D. Jie, J. Wang, H. Zhang, and G. Wang, "Channel capacity of $60 \mathrm{GHz}$ wireless communication systems over indoor line ofsight and non-line-of-sight channels," in Proceedings of the 6th International Conference on Wireless Communications, Networking And Mobile Computing (WiCOM'10), pp. 1-4, Chengdu, China, September 2010.

[18] D. Gesbert, M. Shafi, D. S. Shiu, P. J. Smith, and A. Naguib, "From theory to practice: an overview of MIMO space-time coded wireless systems," IEEE Journal on Selected Areas in Communications, vol. 21, no. 3, pp. 281-302, 2003.

[19] E. Telatar, Capacity of Multi Antenna Gaussian Channels, Technical Memorandum, AT\&T Bell Laboratories, 1995. 
[20] R. Flickenger, "Wireless Networking in the Developing World," University Press of Florida, 2009.

[21] ITU, Propagation Data and Prediction Methods for the Planning of Indoor Radio Communication Systems and Radio Local Area Networks in the Frequency Range 900MHz to $100 \mathrm{GHz}$, Recommendation ITU-R P.1238-7, 2012.

[22] ITU, Sharing between 50/40 GHz Geostationary Networks and Non Geostationary System, Recommendation ITU-R S.2462-0, 2019.

[23] ITU, Technical Feasibility of IMT in Band above 6GHz, ITU-R M.2376-0, 2015.

[24] Ericsson, "Mini-Link 6363 Data Sheet,” 2017, https://tele-a.ru/ wp-content/uploads/2017/07/6363-datasheet.pdf.

[25] Ericsson, "Mini-Link 6352 Data Sheet," 2015, https://tele-a.ru/ wp-content/uploads/2015/09/6352-DS.pdf.

[26] A. M. Saman, M. Cheffena, M. Mohamed, M. H. Azmi, and A. Y. Ai, "Statistical analysis of rain at millimeter waves in tropical area," IEEE Access, vol. 8, pp. 51044-51061, 2020.

[27] H. R. Gauge, "Rainfall data logging system," 2016, https://www .onsetcomp.com/products/data-loggers/rg3/. 